\title{
A THz Single-Polarization-Single-Mode (SPSM) Photonic Crystal Fiber Based on Epsilon-Near-Zero Material \\ Tianyu Yang ${ }^{\mathrm{a}}$, Can Ding ${ }^{\mathrm{a}}$, Richard W. Ziolkowski ${ }^{\mathrm{a}}$ and Y. Jay, Guo ${ }^{\mathrm{a}}$ \\ ${ }^{a}$ Global Big Data Technologies Centre (GBDTC), University of Technology Sydney (UTS), Sydney 2007, AU
}

\begin{abstract}
To overcome the crosstalk happening between two degenerately fundamental modes of a fibre in Terahertz (THz) regime, a novel photonic crystal fiber (PCF) that yields a wide range of single-polarization-single-mode (SPSM) propagation with large loss differences (LDs) is designed. The method used to realize this SPSM PCF is to deposit an epsilon-near-zero (ENZ) material in four selected air holes in the cladding, which ends up with four ENZ rings. These ENZ rings introduce significant LDs between the wanted (X-polarized) and unwanted (Y-polarized and high order) modes. Extensive simulation results demonstrate that the LDs between the wanted and unwanted modes vary with the thickness of ENZ rings. With a very short length $(4 \mathrm{~cm})$ of the proposed PCF, pure SPSM propagation, i.e., the unwanted modes are $20 \mathrm{~dB}$ lower than the wanted mode, can be achieved from 1 to $1.2 \mathrm{THz}$.
\end{abstract}

Keywords: Epsilon-near-zero (ENZ); Fiber characterization; Photonic crystal fiber (PCF); Single-polarization-singlemode (SPSM)

\section{INTRODUCTION}

Polarization maintaining photonic crystal fibers (PM PCF) have been widely used for applications like precision optical instruments and sensors ${ }^{1}$. Generally. there are two types of PM PCFs, i.e., highly birefringent PCF (HB PCF) and singlepolarization-single-mode (SPSM) PCF. To date, various HB PCFs with a birefringence varying from 0.001 to 0.1 have been reported ${ }^{2,3}$. However, the reported THz SPSM PCFs are much less.

There are two methods to realize SPSM PCF. A THz SPSM PCF based on index matching technique is reported ${ }^{4}$, which achieves a SPSM operation in a wide frequency range from 1.67 to $1.8 \mathrm{THz}$. However, the loss difference (LD) between the wanted and unwanted modes are not large enough, i.e., the loss factors are $0.004 \mathrm{~dB} / \mathrm{m}$ and $3.6 \mathrm{~dB} / \mathrm{m}$, respectively. Another method is based on manipulating the asymmetry of the structure to make the cut-off frequencies of two polarized modes different. Therefore, only the higher index mode is allowed to propagate in the fiber core ${ }^{5}$ within the range of these two cut-off frequencies.

In this paper, a THz PCF with circular air holes is developed to achieve SPSM operation. The higher LDs between the wanted (X-polarized) and unwanted (Y-polarized and higher order) modes are realized by depositing the lossy epsilonnear-zero (ENZ) material in the four selected air holes. By tuning the thickness of the ENZ rings, one can control the SPSM bandwidth and LDs between the wanted and any other unwanted modes. The simulation results demonstrate that with proper design the proposed PCF enables SPSM propagation across a wide bandwidth of $0.2 \mathrm{THz}$ where the LDs are above $5 \mathrm{~dB} / \mathrm{cm}$. This means that a pure X-polarized (XP) mode can be attained after propagating several centimeters length within the PCF. All of the simulations in this work were conducted by the COMSOL Multiphysics commercial software package.

\section{CONFIGURATION AND SIMULATION RESULTS}

Cross-sectional view of the proposed THz SPSM PCF configuration is presented in Figure 1a. All the air holes in the cladding share the same diameter $d_{1}$, expect the two air holes alongside the core, which have the diameter $d_{2}$. The lattice constant $\Lambda$ is the distance between any two adjacent holes in the cladding. A perfectly matched layer with a thickness of $12 \%$ of the whole diameter of the PCF is set concentrically on the outside of the cladding. ENZ material is deposited in four selected air holes through high-pressure chemical deposition ${ }^{6}$, resulting in ENZ rings highlighted by the red color in Figure 1. The thickness of these rings is $t$, which can be controlled by deposition time. In this work, we selected high resistivity silicon as the background material because of its great properties, e.g., low bulk material absorption loss < 
$0.015 \mathrm{~cm}^{-1}$ from $0.1-1.5 \mathrm{THz}$ and stable refractive index $3.42^{7}$. Naturally existing materials or metamaterials ${ }^{8}$ can be obtained with ENZ properties. According to the data of the reported ENZ materials, we set to be a constant of $n_{\text {enz }}=0.22$ $-0.33 j$ for the refractive index of the ENZ material used in this work.

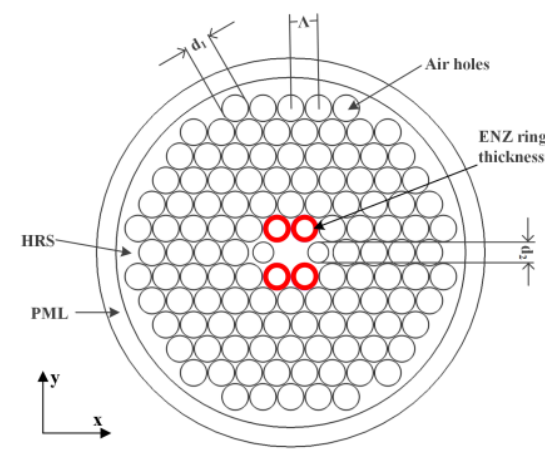

(a)

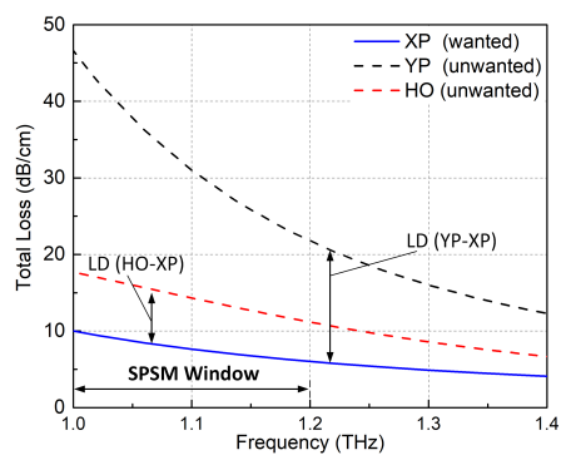

(b)

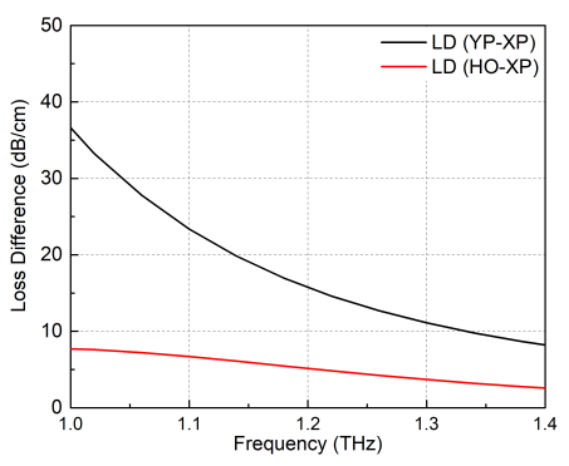

(c)

Figure 1. (a) Cross sectional view of the proposed SPSM PCF. (b) TLs and (c) LDs of the proposed PCF.

Figure $1 \mathrm{~b}$ exhibits the total loss (TL) of each mode, which mainly contains confinement loss and effective material loss. The LDs between the XP and other unwanted modes are plotted in Figure 2c. As shown in the figure, the XP mode has the lowest loss, while the Y-polarized (YP) and high order (HO) modes have larger loss factors. Moreover, the YP mode has the largest loss factor because with the proposed configuration YP mode has highest E-field ratio distributed in the lossy ENZ rings, thus, highest loss. The achieved SPSM band is $0.2 \mathrm{THz}$ from 1 to $1.2 \mathrm{THz}$, where the TL of XP mode is acceptable (below $10 \mathrm{~dB} / \mathrm{cm}$ ) and the LDs are above $5 \mathrm{~dB} / \mathrm{cm}$.

\section{CONCLUSION}

This paper demonstrated a novel PCF design that presents a wide bandwidth of SPSM propagation and large LDs between the XP and other modes. With the investigation, the PCF yields a SPSM operation window from 1 to $1.2 \mathrm{THz}$ and large LDs $>5 \mathrm{~dB} / \mathrm{cm}$, which means that a pure XP state would be obtained after propagating very short distance within the fiber. The proposed THz SPSM PCF would be promising in polarization sensitive systems ${ }^{9}$.

\section{REFERENCES}

[1] P. St. J. Russell, “Photonic-Crystal Fibers,” J. Lightw. Technol. 24(12), 4729-4749 (2006).

[2] S. Atakaramians, S. A. Vahid, H. Ebendorff-Heidepriem, M. Nagel, B. M. Fischer, D. Abbott, and T. M. Monro, "THz porous fibers: design, fabrication and experimental characterization," Opt. Express 17(16), 14053-14062 (2009).

[3] T.-Y. Yang, E. Wang, H. Jiang, Z. Hu, and K. Xie, "High birefringence photonic crystal fiber with high nonlinearity and low confinement loss," Opt. Express 23(7), 8329-8337 (2015).

[4] Y. Hou and G. Wang, "Broadband tunable single-mode single-polarization fiber," Opt. Comm., 351, 91-95 (2015).

[5] D. Lu and J. Liu, "Broadband single-polarization single-mode operation in photonic crystal fibers with hexagonally latticed circular airholes," J. Light. Technol., 34(10), 2452-2458 (2016).

[6] M. A. Schmidt, A. Argyros, and F. Sorin, "Hybrid optical fiber - an innovative platform for in-fiber photonic devices," Adv. Optical Mater. 4(1), 13-36 (2016).

[7] J. Dai, J. Q. Zhang, W. L. Zhang, and D. Grischkowsky, "Terahertz time-domain spectroscopy characterization of the far-infrared absorption and index of refraction of high-resistivity, float-zone silicon," Opt. Soc. Am. B 21(7), 1379-1386 (2004).

[8] N. Engheta and R. W. Ziolkowski, [Metamaterials: Physics and Engineering Explorations], Wiley, 2006.

[9] T.-Y. Yang, C. Ding, R. W. Ziolkowski, and Y. J. Guo, "Circular hole ENZ photonic crystal fibers exhibit high birefringence," Opt. Express 26(13), 17264-17278. 